\title{
A hybrid technique to address exposure keratopathy secondary to facial nerve paresis: a combination of a lateral tarsorrhaphy and lateral wedge resection
}

Roxana Fu, M.D., ${ }^{1,2,3}$ - rfu5@jhmi.edu

Nicholas Moore, M.D. ${ }^{2}$ - mooreni@iupui.edu

Taha Z. Shipchandler, M.D., F.A.C.S. ${ }^{4}$ - tshipchandler@hotmail.com

Jonathan Y. Ting, M.D. ${ }^{4}$ - joting@iupui.edu

Travis Tollefson, M.D., M.P.H., F.A.C.S. ${ }^{5}$ - rfk82@mail.umkc.edu

Cyrus Rabbani, M.D. ${ }^{4}$ - chopfuey87@gmail.com

Meena Moorthy ${ }^{6}$ - mmoorthy@indiana.edu

William R. Nunery, M.D., F.A.C.S. ${ }^{1,2,3}$ - mathieu113@hotmail.com

H.B. Harold Lee, M.D., F.A.C.S. ${ }^{1,2,3}$ - huibae@gmail.com

${ }^{1}$ Department of Ophthalmology and Visual Sciences, University of Louisville, $301 \mathrm{E}$.

Muhammad Ali Blvd, Louisville, Kentucky 40202

${ }^{2}$ Department of Ophthalmology, Indiana University, 1160 W. Michigan St, Indianapolis, Indiana 46202

${ }^{3}$ Oculofacial and Orbital Surgery, 201 Pennsylvania Parkway Ste 225, Indianapolis, Indiana 46280

${ }^{4}$ Department of Otolaryngology, Indiana University, 1130 W. Michigan Street, Suite 400, Indianapolis, Indiana 46202

${ }^{5}$ Department of Otolaryngology, University of California, Davis, 2521 Stockton Blvd, Suite 6201, Sacramento, California 95817

${ }^{6}$ Indiana University School of Medicine, 420 University Blvd, Indianapolis, Indiana 46202

Corresponding Author:

H.B. Harold Lee, MD

Oculofacial Plastic and Orbital Surgery

201 Pennsylvania Parkway

Suite 225

Indianapolis, IN 46280

Phone: $317-573-1000$

Email: huibae@gmail.com

This is the author's manuscript of the article published in final edited form as:

Fu, R., Moore, N., Shipchandler, T. Z., Ting, J. Y., Tollefson, T., Rabbani, C., ... \& Lee, H. H. (2018). A hybrid technique to address exposure keratopathy secondary to facial nerve paresis: A combination of a lateral tarsorrhaphy and lateral wedge resection. American journal of otolaryngology. 
Key words: lower eyelid laxity; facial nerve paresis; exposure keratopathy

Word Count: Total (excluding abstract and references): 1,128 / Abstract: 163

Acknowledgments: All authors listed made a substantial contribution to the study design and acquisition and interpretation of the data. Each author participated in drafting or revising the manuscript and approved submission of this version for publication. Also there are no conflicts of interest to report from any author for this study.

Funding: Indiana University Department of Ophthalmology is supported by Research to Prevent Blindness, New York, NY. However, this organization did not participate in the study design, collection, analysis and interpretation of data, writing of the report, or decision to submit this article for publication. 


\section{ABSTRACT}

Purpose: To present the results of treating combined lower eyelid laxity, retraction and midface descent secondary to facial nerve weakness with a hybrid surgical procedure.

Materials and Methods: A retrospective analysis of patients from January 2015 to January 2017 who underwent a hybrid surgical technique for the treatment of corneal exposure secondary to facial nerve paresis with a single surgeon was performed. Age, gender, and presence of exposure symptoms were recorded pre-operatively. Outcomes assessed included improvement of lower eyelid laxity and position, operative complications, and post-operative symptomatic relief.

Results: A total of 11 patients underwent unilateral eyelid surgery. All patients had symptomatic relief and good functional outcomes defined as improvement in eyelid laxity, lower eyelid position, and objective corneal exposure. No cases required reoperation during an average follow up of 174.5 days.

Conclusions: Combining portions of a tarsorrhaphy and lateral wedge resection technique is a simple and effective procedure to improve lower eyelid position and limit corneal exposure secondary to facial nerve paresis. 


\section{Introduction:}

Malposition of the lower eyelid due to facial nerve weakness may be exacerbated by multiple factors, including involutional changes such as horizontal eyelid laxity, lateral canthal tendon laxity, and midface descent.[1,2] Additional considerations include a negative vector configuration and unopposed lower eyelid retractors.[1] Each factor has its own unique challenges and can be addressed by various surgical techniques. These considerations directly contribute to the static factors that increase interpalpebral fissure distance and worsen the exposed ocular surface area. Dynamic considerations addressed by improved initiation of blink such as eyelid weights and springs are not addressed in many surgical techniques. We present our experience with a modified hybrid technique combining a lateral tarsorrhaphy and lateral wedge resection to improve the lower eyelid position and decrease corneal exposure.

\section{Material and methods:}

After approved by the Indiana University Institutional Review Board, a retrospective chart review was conducted evaluating patients undergoing the hybrid technique by a single surgeon (HBL) from January 2015 to January 2017. Patients were identified with CPT codes 67917 (ectropion repair), 67882 (permanent tarsorrhaphy) and ICD 10 code G51.0 (bell's palsy) and S04.50XA (injury of facial nerve). Medical records of the patients who met these criteria were reviewed to obtain data including age, gender, presence of pre-operative exposure symptoms, post-operative lower eyelid laxity and position, operative complications, post-operative symptomatic relief, and length of follow up.

Surgical Method: 
Corneal protectors are placed in both eyes and the entire lower eyelid and lateral $1 / 3$ of the upper eyelid on the operative side are infiltrated with local anesthetic. A 15 blade and iris scissors are used to perform a lateral canthotomy [Figure 1a,b] and iris scissors are then used to perform the lateral inferior cantholysis [Figure 1c]. A 5-7 $\mathrm{mm}$ incision is made with a 15 blade at the grey line of the lateral most margin of the upper eyelid [Figure 1d]. A full-thickness triangular segment of the lateral eyelid is excised after determining the amount to be removed by approximating the cut edge of the lower lid to the orbital rim [Figure 1e]. A 5-0 polygalactin suture on a P3 needle is passed vertically through the lower border of the lateral tarsus of the lower eyelid and exiting at the lateral cut edge of the tarsus at the margin [Figure 1f]. The needle is then passed through the medial aspect of the upper lid margin incision and retrieved at the superior skin edge of the lateral canthotomy [Figure $1 \mathrm{~g}, \mathrm{~h}$ ]. The needle is then passed through the periosteum of the internal lateral orbital rim at the level of the insertion of the lateral canthal tendon [Figure 1i]. The skin incisions are closed using interrupted 5-0 fast absorbing gut suture. All patients also underwent lower eyelid recession and extirpation of the retractors as described by Compton et al. [2] and upper eyelid gold weight placement.

\section{Results:}

The study identified twelve patients with unilateral lower eyelid repair, for a total of twelve lower eyelids. One patient was excluded secondary to lack of follow up. Of the remaining eleven patients, the mean age was 58.1 years (range 32-77 years); 7 were men and 4 were women. The average follow up time was 174.5 days (range 7 to 560 days). All patients were noted to have improvement of their lower eyelid laxity and position. None of the patients required reoperation during the course of this study. No 
patients reported dissatisfaction with their post-operative appearance. All patients experienced subjective improvement of exposure symptoms and objective improvement of their corneal exposure on exam. No complications were encountered.

\section{Discussion:}

Facial nerve paresis patients face a variety of challenges that contribute to exposure keratopathy, including lower eyelid laxity, eyelid retraction, lagophthalmos, midface descent, and decreased blink. The use of a tarsorrhaphy as a single procedure to reduce exposure keratopathy is common, with many variations including permanent lateral tarsorrhaphies or modifications of a tarsoconjunctival flap.[3] A tarsorrhaphy alone is subject to variable cosmetic outcomes and does not address lower eyelid laxity. Considerations such as eyelid weights and springs address the dynamic aspect of initiating blink and decreasing the vertical interpalpebral fissure distance.[4-5] All our patients underwent gold weight placement. Additionally, all our patients underwent lower eyelid retraction recession and extirpation as described by Compton et al.[2]

Most techniques described for lower eyelid tightening and treating lateral canthal tendon laxity involve anchoring the tarsus to the orbital rim.[6-10] The lateral tarsal strip was first described in 1979 by Richard L. Anderson, and has been used extensively to treat both eyelid laxity, malposition, and lateral canthal tendon laxity.[11] In 2009, Anderson further modified the lateral tarsal strip to include a mini-tarsorrhaphy, to address upper eyelid overhang and lower eyelid imbrication in cases involving concurrent upper eyelid laxity.[12] In 2010, Igal Leibovitch described using a singlestitch lateral wedge technique to address lower eyelid entropion.[8] The lateral wedge resection forgoes the traditional canthotomy cantholysis and utilizes a full thickness 
lateral incision through the lower eyelid to perform an inferior cantholysis. After a full thickness triangular wedge resection of the lateral aspect of the lower eyelid is performed, the tarsus is reattached to the inferior crus of the lateral canthal tendon with a single suture that is passed vertically through the lower cut edge of the tarsus. Our technique combines addition of the upper eyelid tarsorrhaphy, traditional canthotomy cantholysis, and orbital rim fixation with the simplicity of the single-stitch lateral wedge resection.

In patients with a negative vector and midface descent, tightening the lower eyelid does not improve the factors creating eyelid ectropion and retraction. By creating an upward angulation while also improving lower lid laxity, the lower lid position can be markedly improved in this specific cohort of patients. This technique also prevents the cosmetic problems of a traditional permanent tarsorraphy by maintaining a sharp lateral canthal angle and minimizing lateral phimosis. Compared to the traditional lateral tarsal strip, this technique confers a greater surface area of coverage due to higher approximation onto the upper eyelid. In the reduced schematic eyelid aperture, the additional surface area covered can be calculated by subtracting $30 \mathrm{~mm}^{2}$ (the surface area of a triangle) [Figure 2] from $78.5 \mathrm{~mm}^{2}$ (the fraction of the surface area of a circle) [Figure 3], leaving a theoretical $48.5 \mathrm{~mm}^{2}$ of additional area covered with the hybrid technique.

\section{Conclusions:}

The authors find that this hybrid technique is best utilized in patients with horizontal eyelid laxity complicated by midface descent and is a useful adjunct to upper eyelid weight placement and lower eyelid retraction extirpation/recession for patients with 
facial nerve paresis. The addition of attaching the cut edge of the lower eyelid to the upper eyelid creates a new, sharp lateral canthal angle and prevents upper eyelid overhang and imbrication of the lower eyelid. Furthermore, this improves the static exposure in this patient population and addresses their poor involuntary blink rate and incomplete lid closure. 


\section{References:}

[1]. Hahn S, Desai SC. Lower Lid Malposition: Causes and Correction. Facial plastic surgery clinics of North America 2016;24:163-71.

[2]. Compton CJ, Clark JD, Nunery WR, Lee HB. Recession and Extirpation of the Lower Eyelid Retractors for Paralytic Lagophthalmos. Ophthalmic plastic and reconstructive surgery 2015;31:323-4.

[3]. Sufyan AS, Lee HB, Shah H, Nunery WR, Kokoska MS, Shipchandler TZ. SingleStage Repair of Paralytic Ectropion Using a Novel Modification of the Tarsoconjunctival Flap. JAMA Facial Plast Surg. 2014;16:151-2

[4]. Seiff SR, Sullivan JH, Freeman LN, Ahn J. Pretarsal fixation of gold weights in facial nerve palsy. Ophthalmic plastic and reconstructive surgery 1989;5:104-9.

[5]. Demirci H, Frueh BR. Palpebral spring in the management of lagophthalmos and exposure keratopathy secondary to facial nerve palsy. Ophthalmic plastic and reconstructive surgery 2009;25:270-

[6]. Flowers RS. Canthopexy as a routine blepharoplasty component. Clinics in plastic surgery 1993;20:351-65.

[7]. Hesse RJ. The tarsal sandwich: a new technique in lateral canthoplasty. Ophthalmic plastic and reconstructive surgery 2000;16:39-41.

[8]. Leibovitch I. Lateral wedge resection: a simple technique for repairing involutional lower eyelid entropion. Dermatologic surgery: official publication for American Society for Dermatologic Surgery [et al] 2010;36:1412-8.

[9]. Ousterhout DK, Weil RB. The role of the lateral canthal tendon in lower eyelid laxity. Plastic and reconstructive surgery 1982;69:620-3.

[10]. Rees TD. Prevention of ectropion by horizontal shortening of the lower lid during blepharoplasty. Annals of plastic surgery 1983;11:17-23.

[11]. Anderson RL, Gordy DD. The tarsal strip procedure. Archives of ophthalmology (Chicago, III : 1960) 1979;97:2192-6.

[12]. Vagefi MR, Anderson RL. The lateral tarsal strip mini-tarsorrhaphy procedure. Archives of facial plastic surgery 2009;11:136-9. 
Figure Legends:

Figure 1 Surgical Method: A 15 blade (a) followed by iris scissors (b) are used to perform a lateral canthotomy. Iris scissors are used to perform the lateral inferior cantholysis (c). A 5 to $7 \mathrm{~mm}$ incision is made with a 15 blade at the grey line of the lateral most margin of the upper eyelid (d). A full-thickness triangular segment of the lateral eyelid is excised after determining the amount to be removed by approximating the cut edge of the lower lid to the orbital rim (e). A 5-0 polygalactin suture on a P3 needle is passed vertically through the lower border of the lateral tarsus and exiting at the lateral cut edge of the tarsus at the margin ( $f)$. The needle is then passed through the medial aspect of the upper lid margin incision ( $\mathrm{g}$ ) and retrieved at the superior skin edge of the lateral canthotomy (h). The needle is then passed through the periosteum of the internal lateral orbital rim at the level of the insertion of the lateral canthal tendon (i). The final appearance of the eyelids (j).

Figure 2 Surface Area (SA) of Lateral Tarsal Strip (LTS): $30 \mathrm{~mm}$ average horizontal palpebral fissure length (base $(B)$ ), multiplied by $2 \mathrm{~mm}$ average height $(\mathrm{H})$ of the lateral canthus to the medial canthus, divided in half to calculate the relative theoretical surface area covered by a traditional lateral tarsal strip.

Figure 3 Surface Area (SA) of the hybrid technique: The degree measured at the medial canthus divided by 360 degrees, multiplied by the surface area of a circle in which $30 \mathrm{~mm}$ is the radius (r), to calculate the relative theoretical surface area covered by the hybrid technique. 


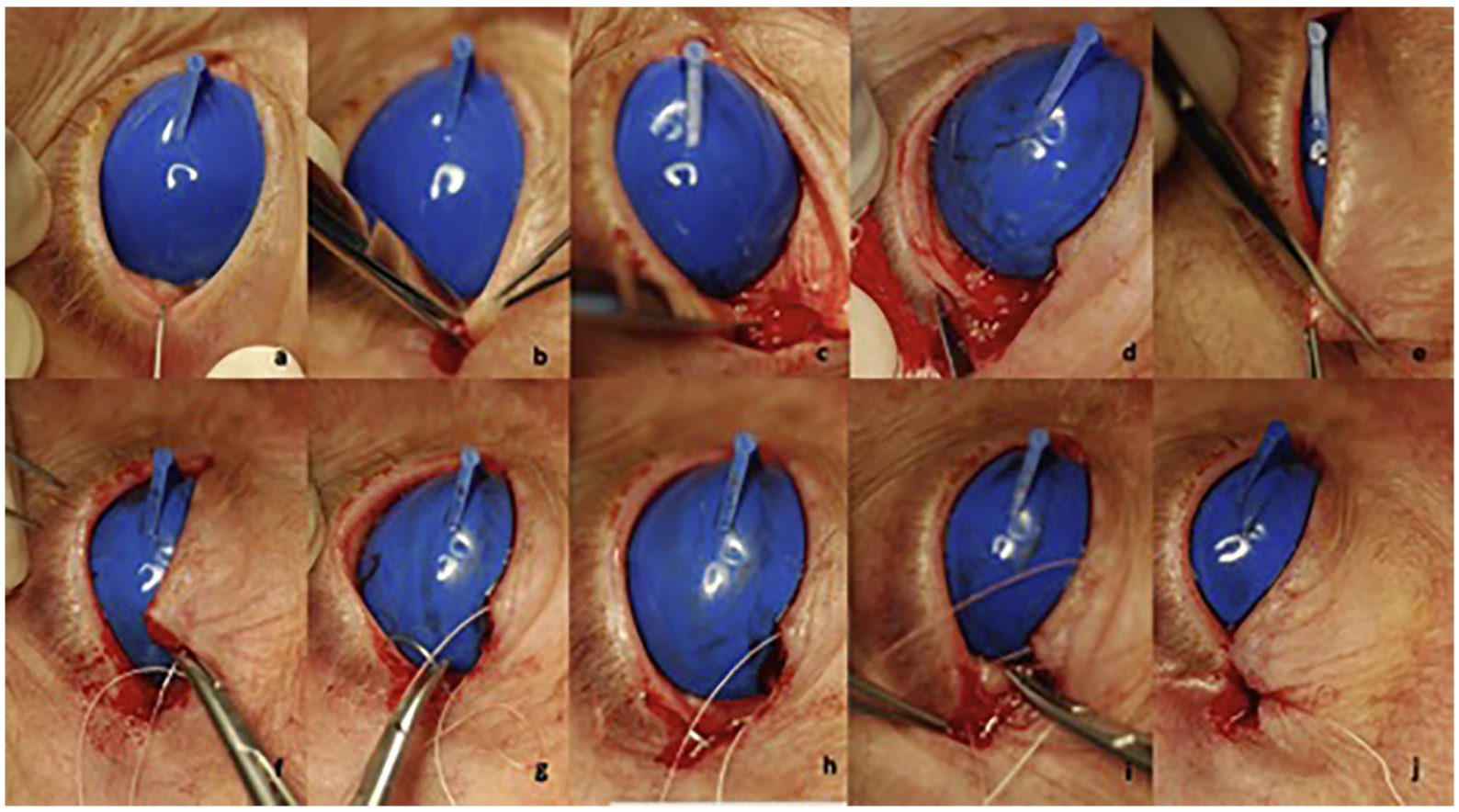

Figure 1 
Upper Eyelid

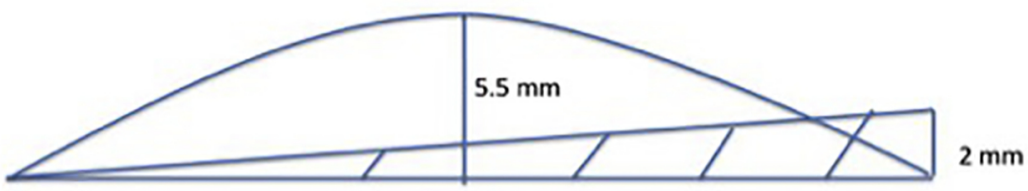

$30 \mathrm{~mm}$

Medial

Lateral

Canthus

Canthus

Lower Eyelid

SA LTS $=(B)(H) / 2=(30 \mathrm{~mm})(2 \mathrm{~mm}) / 2=30 \mathrm{~mm}^{2}$

Figure 2 


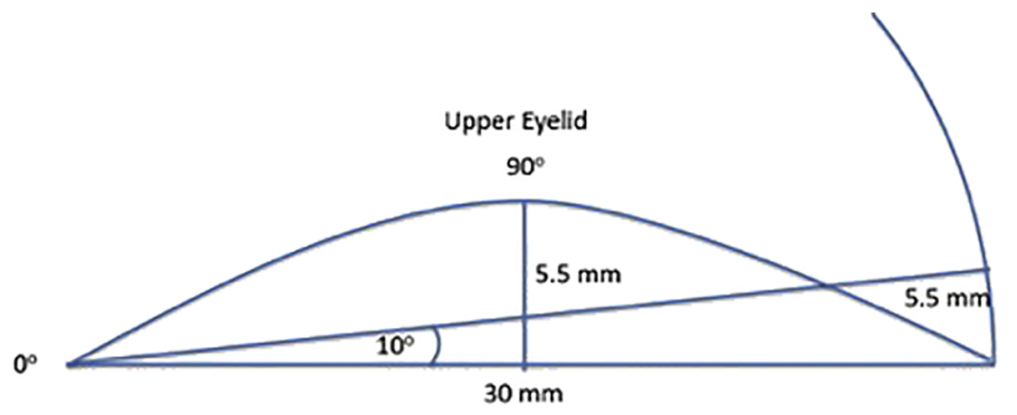

Medial

Canthus

Lower Eyelid

Lateral

Canthus

SA hybrid $=$ Degrees at medial canthus $x \pi r^{2}$

$=10 / 360(\pi)(30 \mathrm{~mm})^{2}=78.5 \mathrm{~mm}^{2}$

Figure 3 